\title{
Visible-Light-Absorbing Potassium Niobate-Titanate-Molybdate Ferroelectrics
}

\author{
Or Shafirø, ${ }^{1}$ Yang Bai $\odot,{ }^{2}$ Jari Juuti $\odot,{ }^{2}$ and Ilya Grinberg ${ }^{1, *}$ \\ ${ }^{1}$ Department of Chemistry, Bar-Ilan University, Ramat Gan 529002, Israel \\ ${ }^{2}$ Microelectronics Research Unit, Faculty of Information Technology and Electrical Engineering, \\ University of Oulu, FI-90014 Oulu, Finland
}

(Received 2 August 2020; revised 14 September 2020; accepted 15 September 2020; published 28 October 2020)

\begin{abstract}
The interactions of ferroelectric (FE) perovskite oxides $\left(\mathrm{ABO}_{3}\right)$ with light are increasingly being studied for different applications, such as photovoltaics and optoelectronics. The combination of different cations at the $A$ and $B$ sites to form solid solutions allows tuning of the material's properties and, most importantly, the band gap $\left(E_{g}\right)$, which sets the wavelength range of light absorption. Classic FE perovskite oxides, such as $\mathrm{BaTiO}_{3}, \mathrm{KNbO}_{3}$, and $\mathrm{PbTiO}_{3}$, exhibit $E_{g}>3 \mathrm{eV}$, which limits their implementation in visible-lightabsorbing devices. Furthermore, the tuning of their $E_{g}$ via a solid solution strategy to a lower $E_{g}$ range is limited by the requirement for the presence of a $d^{0}$ metal at the $B$ site, which is necessary for the FE distortion, but leads to a larger $E_{g}$. This gives rise to the challenge of decreasing $E_{g}$, while maintaining FE distortion. Here, we use first-principles calculations to explore the FE and optical properties of the $\left(\mathrm{KNbO}_{3}\right)_{x}\left(\mathrm{KTi}_{1 / 2} \mathrm{Mo}_{1 / 2} \mathrm{O}_{3}\right)_{1-x}(\mathrm{KNTM})$ perovskite oxide solid solution. The introduction of $\mathrm{Ti}^{4+}$ and $\mathrm{Mo}^{6+}$ into the parent $\mathrm{KNbO}_{3}$ decreases the $E_{g}$ to about $2.2 \mathrm{eV}$ for $x=0.9$, while preserving or enhancing polarization. Experimental fabrication and characterization show that the obtained KNTM material at $x=0.9$ has an orthorhombic structure at room temperature and a direct gap of $<2.2 \mathrm{eV}$, confirming firstprinciples-based predictions. These properties make KNTM a promising candidate for further studies and applications as a visible-light-absorbing FE material.
\end{abstract}

DOI: 10.1103/PhysRevApplied.14.044052

\section{INTRODUCTION}

Ferroelectric (FE) perovskite $\left(A B \mathrm{O}_{3}\right)$ oxides have been studied for close to 80 years as functional materials in a wide variety of piezoelectric, optoelectronic, and memory applications and are also fundamentally fascinating materials due to the complex interplay of soft and hard degrees of freedom, structural properties, and functional response. Over the last decade, there has been a surge of interest in the study of the interactions of FE with light, particularly for photovoltaic applications [1-3]. Owing to their polar nature, FE materials exhibit the bulk photovoltaic effect (BPVE) that can, in principle, allow a power conversion efficiency above the Shockley-Queisser limit in PV devices, as demonstrated for $\mathrm{BaTiO}_{3}$ (BTO) material with nanoscale electrodes [4]. However, classic FEs, such as $\mathrm{BTO}, \mathrm{KNbO}_{3}(\mathrm{KNO})$, and $\mathrm{PbTiO}_{3}$ (PTO) have high band gaps $\left(E_{g}\right)[5-7]$ that preclude the absorption and utilization of visible light and most of the energy of the solar spectrum. The high $E_{g}$ is due to the $d^{0}$ transition-metal electronic structure necessary for the second-order JahnTeller distortion that drives the off-center displacements of the $B$ cations, which give rise to the FE response. The

\footnotetext{
*ilya.grinberg@biu.ac.il
}

large electronegativity difference between the $d^{0}$ metal and the $\mathrm{O}$ atom means that across-the-gap excitation, which is essentially charge transfer from the $\mathrm{O} 2 p$ states to the metal $d$ states has high energy, leading to large $E_{g}$ for FE materials. Recently, this limitation has been overcome by incorporating non- $d^{0}$-metal states into FE perovskite, first in the KBNNO material [KNO modified by doping with $\mathrm{Ba}\left(\mathrm{Ni}_{0.5} \mathrm{Nb}_{0.5}\right) \mathrm{O}_{3-} \delta$ ] [7] and then in many other works using a variety of compositions [5,6,8-10]. The group of non- $d^{0}$-perovskite oxides also includes compounds for which ferroelectricity is driven by the $A$-site cation lone pair, such as $\mathrm{BiFeO}_{3}(\mathrm{BFO})$. Previous works on relatively low-gap $\left(E_{g} \approx 2.7\right) \mathrm{BFO}$ demonstrated the induction of photocurrent by visible light, exploiting the BPVE to generate photovoltages larger than the band gap [11-14]. However, despite exhibiting a relatively low band gap for an oxide perovskite, the band gap of BFO is within the upper limit of visible-light absorption, preventing it from utilizing most of the visible-light range. Incorporation of $\mathrm{Cr}$ (additional non- $d^{0}$ metal) into $\mathrm{BFO}$ to form $\mathrm{Bi}_{2} \mathrm{FeCrO}_{6}$ reduces the band gap by about $1 \mathrm{eV}$, albeit accompanied by a FE polarization decrease [15], similar to its combination with KNO in KNO-BFO solid solution [16]. In general, the approach of using a non- $d^{0}$ metal to reduce the band gap often involves the formation of oxygen vacancies, loss of 
polarization, sensitivity to synthetic conditions, and weak light absorption due to the dipole-forbidden nature of the across-the-gap transition.

Here, we first use density-functional theory (DFT) calculations to investigate an alternative approach of achieving visible-light absorption in $\mathrm{KNO}$ through inclusion of the highly electronegative $\mathrm{Mo}^{6+}$ cation coupled with the $\mathrm{Ti}^{4+}$ ion at the $B$ site, to form $\mathrm{K}(\mathrm{Nb}, \mathrm{Mo}, \mathrm{Ti}) \mathrm{O}_{3}$ (KNTM), and then validate the computational design by the synthesis and characterization of KNTM ceramics. Our previous computational design studies have shown that $\mathrm{Mo}^{6+}$ substitution into $\mathrm{BaTiO}_{3}$, coupled with the substitution of either $\mathrm{Mg}^{2+}$ at the $B$ site or $\mathrm{Na}+$ at the $A$ site, can lower the band gap to about $2.5 \mathrm{eV}$ [17]. However, this $E_{\mathrm{g}}$ value is still too high for the efficient light absorption necessary in photovoltaic cells. Here, we pursue an alternative strategy of using $\mathrm{KNO}$ as a parent material and using the coupled substitution of $\mathrm{Mo}^{6+}$ and $\mathrm{Ti}^{4+}$ to achieve a low $E_{\mathrm{g}}$ value. This approach preserves the $d^{0}$ nature of the $B$-cation sublattice and its full occupation by the FE-active cations. The high distortion ability of Mo and $\mathrm{Ti}$ [18] also means that a high polarization $(P)$ will be preserved or enhanced in the KNO-based material by coupled Mo and Ti substitution, while the $\mathrm{O} 2 p$-metal $4 d$ across-the-gap excitation is dipole-allowed and is likely to show strong absorption that is favorable for the use of KNTM in thin films. In addition, the KNTM material is also fundamentally interesting because, despite the long history of KNO and its importance for FE and piezoelectric materials, the family of 4+ and 6+-substituted KNO materials is not investigated. Our DFT calculations show that KTNM shows a low direct band gap of about $2.0 \mathrm{eV}$ and an enhanced $P$ compared with that of KNO. We then perform experimental synthesis and characterization to confirm the first-principles predictions, obtaining a FE Mo-substituted perovskite and a KNO-based solid solution with $4+$ and $6+$ substitution. We find that the obtained KNTM materials exhibit good visible-light absorption, with $E_{\mathrm{g}}<2.2 \mathrm{eV}$ and strong $P$, which is encouraging for the further exploration of the synthesis, properties, and applications of $\mathrm{Mo}^{6+}$-based perovskites.

\section{METHODOLOGY}

To computationally investigate how the substitution of KNTM affects the properties of $\mathrm{KNO}, \mathrm{Nb}$ atoms are combined with the Mo and Ti atoms at the $B$ site in a ratio of 7:1 to form $\left(\mathrm{KNbO}_{3}\right)_{0.875}\left(\mathrm{KTi}_{1 / 2} \mathrm{Mo}_{1 / 2} \mathrm{O}_{3}\right)_{0.125}$ and in a ratio of $3: 1$ to form $\left(\mathrm{KNbO}_{3}\right)_{0.75}\left(\mathrm{KTi}_{1 / 2} \mathrm{Mo}_{1 / 2} \mathrm{O}_{3}\right)_{0.25}$, which are referred as $87.5-12.5 \mathrm{KNTM}$ and $75-25 \mathrm{KNTM}$, respectively. For the 75-25KNTM solid solution, the (001), (011), and (111) arrangements are studied, which are named according to the orientation of the vector connecting Ti and Mo. For the 87.5-12.5KNTM solid solution, the (001)- $A$, (001)- $B$, (011)- $A$, (011)- $B,(111)-A$, and (111)- $B$ arrangements are studied, with $A$ and $B$ indicating the two possible ionic positions (see Fig. S1 within the Supplemental Material for schematic descriptions of the arrangements [19]). For each arrangement, different initial displacements are imposed and then full ionic relaxations are performed. To obtain highly accurate band-gap estimates, we perform DFT calculations using hybrid HSE06 all-electron calculations with the FHI-aims code for the 75-25KNTM system. The HSE06 band gaps have an almost constant difference of $1.19 \mathrm{eV}$, on average, from the local-density approximation (LDA) $E_{g}$ values (see Fig. S2 and Table SI within the Supplemental Material [19]). Therefore, a simple addition of this correction to the LDA $E_{g}$ is used to predict highly accurate HSE06 $E_{g}$ values for all studied structures. The ionic relaxations are carried out using the Quantum ESPRESSO (QE) package [20] with LDA [21,22] for the exchange-correlation functional. LDA is well known to underestimate the unit-cell volume and the FE distortion in such a system $[23,24]$. Therefore, we perform calculations using the expected experimental lattice parameters of $75-25 \mathrm{KNTM}$ that are obtained by multiplying the underestimated LDA $75-25 \mathrm{KNTM}$ lattice parameters by the ratio of the parent $\mathrm{KNO}$ experimental lattice parameters and the KNO lattice parameters obtained by LDA calculations. Polarization $(P)$ calculations are performed using the Berry phase method, as implemented in QE.

For the experimental validation of the first-principles predictions, the conventional solid-state route is used to fabricated KTNM ceramics. Starting reactants of $\mathrm{K}_{2} \mathrm{CO}_{3}$ ( $\geq 99 \%$, J. T. Baker), $\mathrm{Nb}_{2} \mathrm{O}_{5}$ (99.9\%, Aldrich Chemistry), $\mathrm{MoO}_{3}$ (99.5\%, Alfa Aesar), and $\mathrm{TiO}_{2}$ (99.9\%, Alfa Aesar) powders are weighed precisely, according to the target stoichiometry, with an electronic balance of $0.01 \mathrm{mg}$ readability and $1 \mathrm{mg}$ accuracy (ES 225SM-DR, Precisa, Switzerland). The amounts of $\mathrm{K}_{2} \mathrm{CO}_{3}, \mathrm{Nb}_{2} \mathrm{O}_{5}, \mathrm{MoO}_{3}$, and $\mathrm{TiO}_{2}$ in the raw mixture of the reactants are $34.57,59.83$, 3.60 , and $2.00 \mathrm{wt} \%$, respectively. The parental composition, $\mathrm{KNO}$, is also fabricated alongside $90-10 \mathrm{KNTM}$ for comparison. The amounts of $\mathrm{K}_{2} \mathrm{CO}_{3}$ and $\mathrm{Nb}_{2} \mathrm{O}_{5}$ are 34.21 and $65.79 \mathrm{wt} \%$, respectively. An extra $5 \mathrm{wt} \%$ of $\mathrm{K}_{2} \mathrm{CO}_{3}$ is added to the starting reactants for both $\mathrm{KNO}$ and 90 $10 \mathrm{KNTM}$ to compensate for the potential volatilization of $\mathrm{K}$ at high temperatures during calcination and sintering. As some reactants, such as $\mathrm{K}_{2} \mathrm{CO}_{3}$ and $\mathrm{Nb}_{2} \mathrm{O}_{5}$, are hygroscopic, all reactants are dried at $220^{\circ} \mathrm{C}$ for over $4 \mathrm{~h}$ before weighing, to ensure the correct stoichiometry. The weighed reactants are mixed in a $\mathrm{ZrO}_{2}$ jar on a planetary ball mill (Pulverisette 6, Fritsch, Germany) with $\mathrm{ZrO}_{2}$ balls ( $3 \mathrm{~mm}$ in diameter) and ethanol as the milling media. After $6 \mathrm{~h}$ of mixing and subsequent drying at $80^{\circ} \mathrm{C}$, a one-step calcination at $725^{\circ} \mathrm{C}$ for $4 \mathrm{~h}$ in air is applied to the reactant mixtures. The calcined powders are milled again for $12 \mathrm{~h}$ following the above-described procedure. After drying at $80{ }^{\circ} \mathrm{C}, 5 \mathrm{wt} \%$ of binder $(5 \mathrm{wt} \%$ polyvinyl alcohol dissolved in deionized water) is added and mixed with 
the powders. The powder-binder mixtures are uniaxially pressed at $90 \mathrm{MPa}$ into disk-shaped green bodies with a diameter of $14.5 \mathrm{~mm}$. Following burning off of the binder at $500{ }^{\circ} \mathrm{C}$, the disks are sintered at $1000^{\circ} \mathrm{C}$ for $4 \mathrm{~h}$ (for $90-10 \mathrm{KNTM}$ ) and at $1040^{\circ} \mathrm{C}$ for $4 \mathrm{~h}$ (for KNO). During sintering, the disks are buried with calcined powders of the same compositions and sealed in an $\mathrm{Al}_{2} \mathrm{O}_{3}$ crucible. It should be pointed out here that both sintering temperatures are very close to the melting temperatures of the corresponding compositions, i.e., $1020^{\circ} \mathrm{C}$ for $90-10 \mathrm{KNTM}$ and $1060^{\circ} \mathrm{C}$ for KNO. The sintered ceramic samples are polished with P1200 silicon carbide abrasive paper to make flat and parallel surfaces for characterization.

XRD (D8 Discover, Bruker, Germany) and UV-tovisible-to-near-infrared (UV-vis-NIR) spectroscopy (Cary 500 Scan, Varian, USA) are used to characterize the structure and light-absorption behavior of the ceramic samples, respectively. The samples are also characterized by differential scanning calorimetry (DSC; STA449, NETZSCH, Germany) to identify the phase transitions. The dielectric properties are measured using an LCR meter (4284A, Hewlett Packard, USA) combined with a sample-stage apparatus (LTS 350, Linkam Scientific Instruments, UK) to control the temperature. The FE properties are tested by a FE evaluation system (Precision LCII, Radiant Technologies Inc., USA). For dielectric and FE characterizations, the samples are coated with Ag electrodes (DT 1402, Heraeus, fired at $600{ }^{\circ} \mathrm{C}$ for $20 \mathrm{~min}$ ) prior to measurements.

\section{RESULTS}

To investigate the influence of $\mathrm{Ti}$ and Mo substitution in $\mathrm{KNO}$, we carry out DFT calculations for the KNTM material. The average of $E_{g}$, total polarization $\left(P_{\text {tot }}\right)$, and DFT energy differences relative to the lowest energy structure $(\Delta E)$ are given in Table I, together with the standard deviations for each of the obtained phases (for information regarding each studied structure see Tables SII and SIII within the Supplemental Material [19]). The $P$ vector
TABLE I. Average of $E_{g}$ (obtained using the HSE06-based correction), $P_{\text {tot }}$, and relative energy (compared within the same composition) for the studied compositions. For each composition, the structures in the same phase are averaged and presented in each row. The " \pm " value indicate standard deviation, and "*” indicates an energetically favorable phase (see Tables SII and SIII within the Supplementary Material [19] for structures used for these averages).

\begin{tabular}{cccc}
\hline \hline Phase & $E_{g}(\mathrm{eV})$ & $P_{\text {tot }}\left(\mathrm{C} / \mathrm{m}^{2}\right)$ & $\Delta E(\mathrm{meV} /$ atom $)$ \\
\hline \multicolumn{4}{c}{$87.5-12.5 \mathrm{KNTM}$} \\
$C$ & $1.93 \pm 0.03$ & 0.00 & $7.48 \pm 0.53$ \\
$T$ & $1.96 \pm 0.30$ & $0.29 \pm 0.03$ & $4.36 \pm 0.82$ \\
$M_{C}$ & $2.61 \pm 0.09$ & $0.37 \pm 0.02$ & $2.17 \pm 0.55$ \\
$O$ & $2.75 \pm 0.22$ & $0.38 \pm 0.05$ & $1.47 \pm 0.99$ \\
$M_{B}{ }^{*}$ & $2.91 \pm 0.03$ & $0.39 \pm 0.04$ & $0.96 \pm 0.60$ \\
$R$ & \multicolumn{3}{c}{ not obtained } \\
$M_{A}$ & not obtained \\
\multicolumn{4}{c}{$75-25 \mathrm{KNTM}$} \\
$C$ & $1.94 \pm 0.03$ & 0.00 & $11.24 \pm 2.05$ \\
$T$ & $2.06 \pm 0.25$ & $0.29 \pm 0.02$ & $6.81 \pm 2.15$ \\
$M_{C}$ & $2.40 \pm 0.02$ & 0.37 & $3.33 \pm 1.41$ \\
$O$ & $2.41 \pm 0.19$ & $0.37 \pm 0.01$ & $5.09 \pm 2.18$ \\
$M_{B}{ }^{*}$ & 2.69 & 0.42 & 0.00 \\
$R$ & 2.59 & 0.40 & 4.83 \\
$M_{A}$ & 2.61 & 0.40 & 3.40 \\
\hline \hline
\end{tabular}

direction is used to determine the obtained phases, and the monoclinic phases $\left(M_{A}, M_{B}\right.$, and $\left.M_{C}\right)$ are named according to Vanderbilt and Cohen [25]. In addition, in Figs. 1 and 2, we plot $\Delta E, P_{\text {tot }}$, and $E_{g}$ as a function of the obtained phase [see Fig. S3 within the Supplemental Material for the change of $E_{g}$ and $P_{\text {tot }}$ as a function of the KNTM content [19] ].

Examination of the energy differences between the arrangements within both compositions [Figs. 1(a) and 2(a)] shows that they are fairly small and are on the same scale as the energy differences in the alloys where the $B$ site arrangement is known to be disordered [26]. Thus, (a)

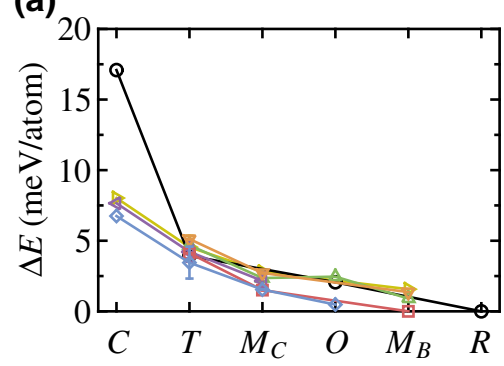

(b)

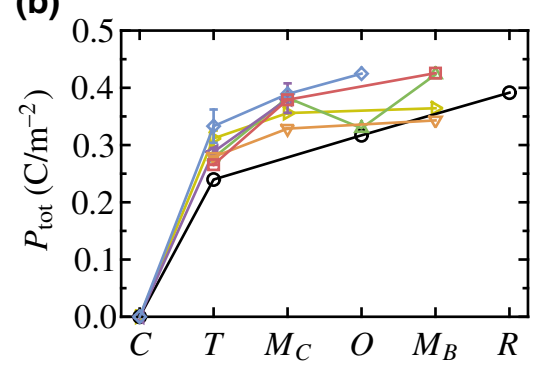

(c)

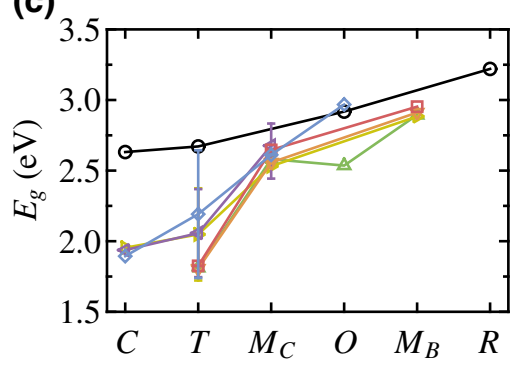

$$
\begin{array}{|llll|}
\hline \square-(001)-A & \Delta-(011)-A & \nabla-(111)-A & 0-\mathrm{KNO} \\
\diamond-(001)-B & \triangleleft-(011)-B & \triangleright-(111)-B & \\
\hline
\end{array}
$$

FIG. 1. Relative energy within composition $\Delta E(\mathrm{a}), P_{\text {tot }}(\mathrm{b})$, and $E_{g}$ (c) of the studied 87.5-12.5KNTM structures and parent KNO. 
(a)

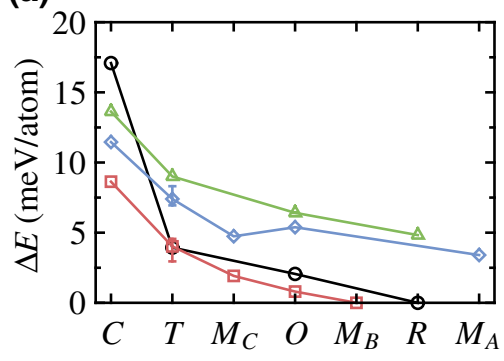

(b)

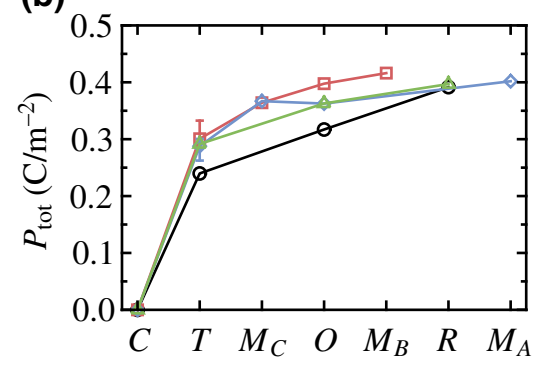

(c)

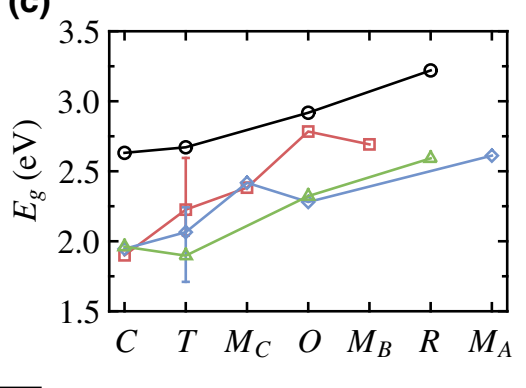

$\mathrm{O}-\mathrm{KNO} \square-(001) \diamond-(011) \Delta-(111)$

FIG. 2. Relative energy within composition $\Delta E$ (a), $P_{\text {tot }}\left(\right.$ b), and $E_{g}$ (c) of the studied 75-25KNTM structures and parent KNO.

it is likely that all three local Mo-Ti arrangements will be present in KNTM, albeit with a greater content of the (100) arrangement compared with that of the (110) and (111) arrangements. The low energy difference is likely to be due to the small deviation of the Mo and Ti formal charges from the +5 average charge of $\mathrm{Nb}$ and the KNTM $B$ site. Examination of $\Delta E$ between different phases shows that, unlike $\mathrm{KNO}$, which prefers the $R$ phase at $0 \mathrm{~K}$, in KNTM, $O$ (orthorhombic) and $M_{B}$ (monoclinic) phases are preferred, and all phases are close in energy. Our DFT calculations show $P$ values of 0.43 and $0.42 \mathrm{C} / \mathrm{m}^{2}$ for the obtained lowest-energy structures of $87.5-12.5 \mathrm{KNTM}$ and $75-25 \mathrm{KNTM}$, respectively, compared with $0.37 \mathrm{C} / \mathrm{m}^{2}$ obtained for the parent KNO material [Figs. 1(b) and 2(b)]. Thus, as expected, the substitution of highly FEactive Ti and Mo cations enhances $P$. The relaxed KNTM structures show no octahedral tilting. Owing to the correlation between polarization and the Curie temperature $\left(T_{C}\right)$ $[27,28]$, it is likely that the phase transitions in KNTM will be shifted to higher temperature than those in KNO. Examination of the $E_{g}$ data shows that Ti-Mo substitution lowers the gap between different ferroelectric phases relative to that of the parent material, as observed in Figs. 1 (c) and 2(c). For 75-25KNTM, $E_{g}$ values of $2.59 \mathrm{eV}$ for the $R$ phase, $(2.41 \pm 0.19) \mathrm{eV}$ for the $O$ phase, and $(2.06 \pm 0.25) \mathrm{eV}$ for the $T$ phase are obtained, on average (Table I). For the 87.5-12.5KNTM, band-gap values of $(2.75 \pm 0.22)$ and $(1.96 \pm 0.30) \mathrm{eV}$ are obtained for the $O$ and $T$ phases, respectively, on average. No $R$ phase is found in this composition; however, the $M_{B}$ phase (which is the most similar to the $R$ phase in polarization direction) is found to have a band gap of $(2.91 \pm 0.03) \mathrm{eV}$, on average. These values are lower than the corresponding $E_{g}$ of the parent $\mathrm{KNO}(3.33,3.1$, and $2.71 \mathrm{eV}$ in the $R$, $O$, and $T$ phases, respectively). The relatively small energy difference between the $O$ and $T$ phases suggests that the low approximately $2.0 \mathrm{eV} E_{\mathrm{g}}$ value of the $T$ phase can be obtained by an application of a small compressive strain, if KNTM is grown in thin-film form on an appropriate substrate.
To validate the feasibility of the coupled Mo and Ti substitution and verify the first-principles predictions, experiments are carried out to fabricate and characterize KNTM ceramic samples. The $90-10 \mathrm{KNTM}$ composition is chosen for experimental validation. It should be noted here that efforts to fabricate other compositions, such as 75$25 \mathrm{KNTM}$, have also been made. However, a perovskite structure is not formed in this composition (Fig. S4 within the Supplemental Material [19]); this is most likely to be due to the competing phases that may be promoted by the preference of Mo for a nonperovskite structure (as observed in $\mathrm{MoO}_{3}$ ) and the stability of multiple Mo oxidation states in oxides. For this reason, only $90-10 \mathrm{KNTM}$, as a representative of the KNTM system, is studied experimentally in this work.

Figure 3(a) shows the XRD patterns of the fabricated 90$10 \mathrm{KNTM}$ and KNO ceramics obtained at room temperature. Both 90-10KNTM and KNO form pure orthorhombic perovskite phases. The lattice parameters calculated from Rietveld refinement are also given in Fig. 3(a). Notably, special sintering conditions, as described in Sec. II are necessary to suppress the formation of secondary phases, and thus, to obtain pure perovskite phases. Figures 3(b) and 3(c) show changes to the relative permittivity and dielectric loss at $100 \mathrm{~Hz}-1 \mathrm{kHz}$ with the measurement temperature for 90-10KNTM. Both parameters exhibit a strong dependence on frequency, most likely due to the high leakage of the sample, making it difficult to identify potential phase transitions. In the inset of Fig. 3(b), neither the DSC curve of 90-10KNTM nor that of KNO shows any sign of a ferroelectric-ferroelectric phase transition. A ferroelectric-ferroelectric (i.e., orthorhombic-tetragonal) phase transition, which is expected for KNO [29] at approximately $220^{\circ} \mathrm{C}$, can be observed in Fig. 3(b). However, a similar one could be neither identified from the temperature-dependent permittivity profile nor observed in the DSC curve for 90-10KNTM. To confirm this, XRD is carried out at $30-380^{\circ} \mathrm{C}$, with $50^{\circ} \mathrm{C}$ intervals, and no differences are observed between the XRD patterns measured at different temperatures, apart from the transition 
(a)

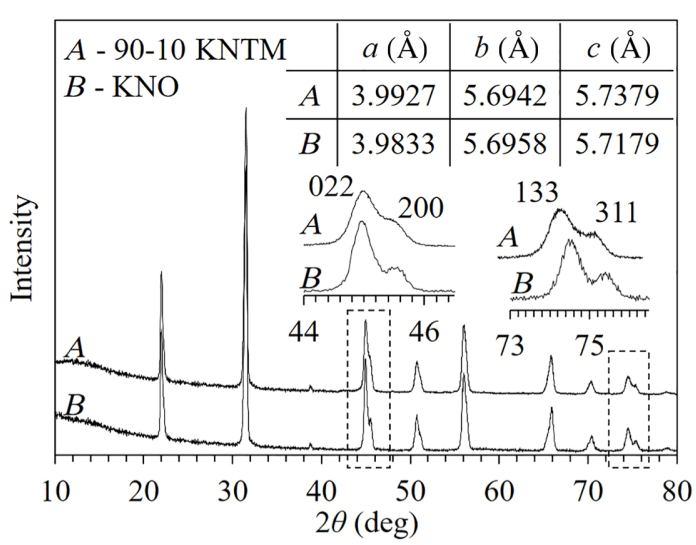

(c)
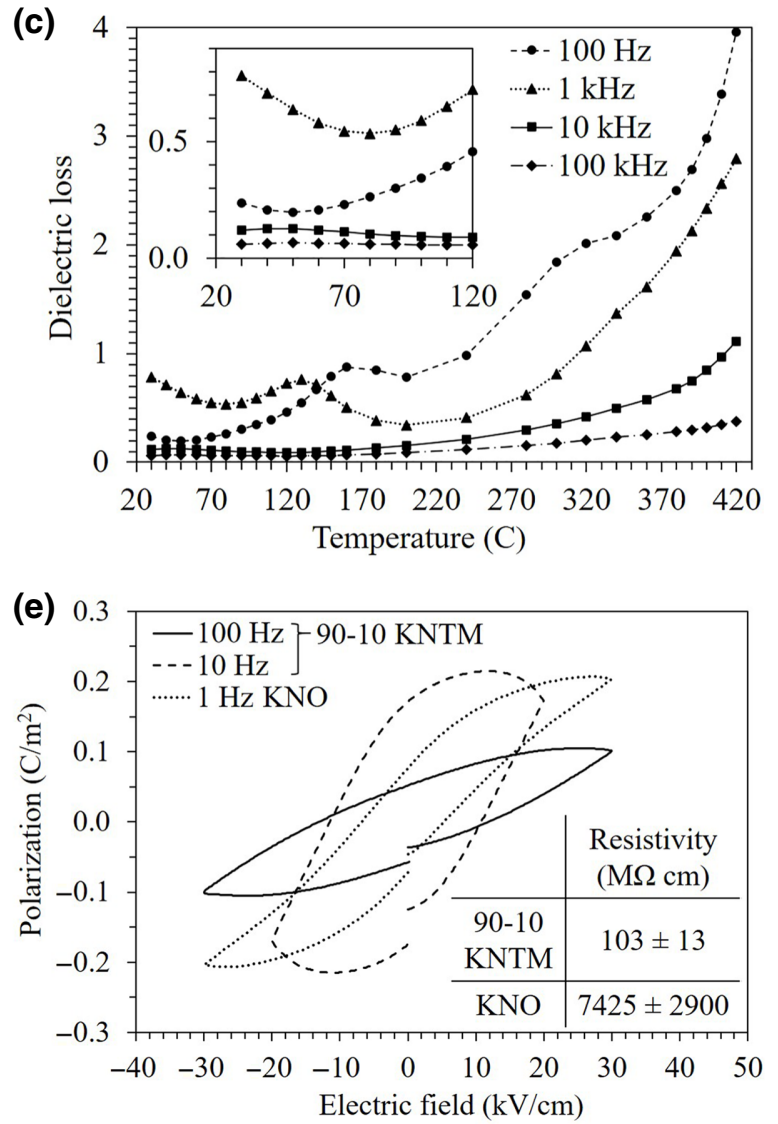

(b)

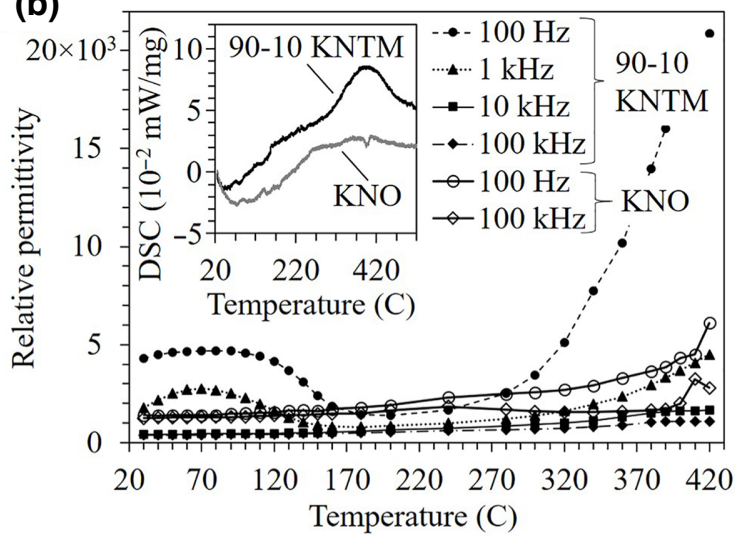

(d)

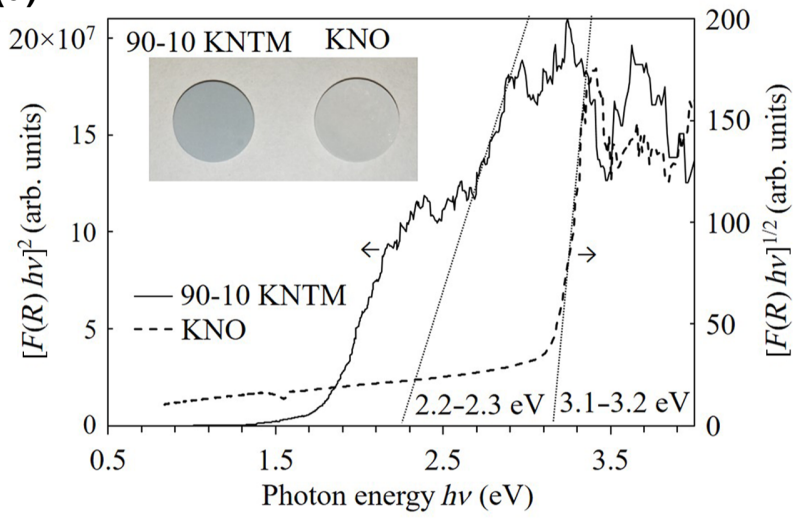

FIG. 3. Characterization of fabricated 90-10KTNM ceramics: (a) XRD pattern obtained at room temperature compared with that of KNO. (b) Dependence of relative permittivity on temperature measured at $100 \mathrm{~Hz}-100 \mathrm{kHz}$; the inset shows the DSC curve compared with those of KNO. (c) Dependence of dielectric loss on temperature measured at $100 \mathrm{~Hz}-100 \mathrm{kHz}$. (d) Tauc relation at room temperature, compared with that of KNO; the inset shows the appearance of the fabricated ceramics. (e) FE hysteresis loops measured in the dark, at room temperature, and with different frequencies compared with that of KNO.

toward the cubic phase near $T_{C}$. The disappearance of the orthorhombic-tetragonal phase transition on 90-10KNTM may be attributed to two possible reasons. First, the substitution of Mo and Ti changes the polarization values of the orthorhombic and tetragonal phases with a greater enhancement in the polarization of the orthorhombic phase. This may stabilize the orthorhombic phase and push the temperature of the orthorhombic-tetragonal phase transition higher, close to the tetragonal-cubic phase transition, so that the orthorhombic-tetragonal phase transition may not be easily noticeable. Furthermore, since $\mathrm{Mo}^{6+}$ and $\mathrm{Ti}^{4+}$, which are smaller than $\mathrm{Nb}^{5+}$, are present at the $B$ site, the phase transition may be suppressed by the internal lattice strain, resulting from lattice distortion. An elucidation 
of the origin of this effect requires additional theoretical and experimental study and is outside the scope of this work.

Figure 3(d) shows the Tauc plots for the fabricated 90$10 \mathrm{KNTM}$ and KNO ceramic samples. 90-10KNTM has a lavender color, compared with an ivory color for KNO [see the inset of Fig. 3(d)]. The fitting of $F(R)$ [where $R$ is reflectance, $F(R)=(1-R)^{2} / 2 R$ ] [30] data suggests that 90-10KNTM has a direct $E_{g}$ in the 1.9-2.2 eV range, compared with the indirect $E_{\mathrm{g}}$ of $3.2 \mathrm{eV}$ for KNO (Figs. S5 and S6 within the Supplemental Material [19]). The uncertainty for the precise position of the band edge of KNTM may be due to the presence of mixed phases (which, according to DFT, have different $E_{\mathrm{g}}$ values), stoichiometry fluctuations, or noise in the data due to the use of a thick $(100 \mu \mathrm{m})$ sample. Detailed $E_{g}$ determinations, according to both direct and indirect assumptions, are shown in Fig. S6 within the Supplemental Material [19], where the values of 1.9-2.2 eV direct $E_{g}$ for 90-10KNTM and $3.2 \mathrm{eV}$ indirect $E_{g}$ for $\mathrm{KNO}$ are considered to be reasonable. The reduction of $E_{g}$ is about $1 \mathrm{eV}$, and the measured $E_{g}$ values for both 90-10KNTM and KNO are consistent with the first-principles-based predictions presented above. Bandgap identification using the Tauc fitting method may be affected by factors such as impurity phases and defects. As shown in Fig. 3(a), both 90-10KNTM and KNO ceramics form pure perovskite phases, without noticeable impurities or a secondary phase. Meanwhile, identical amounts of codopants, $\mathrm{Mo}^{6+}$ and $\mathrm{Ti}^{4+}$, and the resultant charge neutrality make the formation of point defects unlikely. Therefore, the band-gap reduction here is considered to be the effect of codoping, rather than the impurity phase or defects.

The spectrum of KNTM in Fig. 3(b) for the direct-bandgap Tauc plot calculation exhibits two linear regions, with the lower one extrapolated to about $1.7 \mathrm{eV}$ and the higher one extrapolated to about $2.1 \mathrm{eV}$. Since the lower gap estimate is much smaller than the value predicted by our DFT calculations and the DFT HSE06 band-gap values are highly accurate, it is likely that the higher value represents the true intrinsic band gap of KNTM, while the lower value may be due to deviations in the stoichiometry of the experimental KNTM material that lead to further lowering of the band gap. Thus, the higher value is likely to be a more accurate representation of the stoichiometric KNTM material designed in our first-principles calculations. The onset of absorption at lower energies $(\sim 1.6 \mathrm{eV})$ may be due to the presence of regions with slight changes in stoichiometry, for example, oxygen or $\mathrm{K}$ vacancies, that will likely affect Mo- $\mathrm{O}$ bonding in KNTM and the energy of the light-absorption onset. The direct absorption of KNTM is favorable for its use in thin films in PV applications due to the good light absorption of the direct-gap materials.

Figure 3(e) shows the polarization-electric field $(P$ $E$ ) hysteresis loops observed for the fabricated ceramic samples. For 90-10KNTM, the loops are frequency dependent. A lower frequency $(10 \mathrm{~Hz})$ tends to lead to a lossy loop, meaning that the maximum polarization is much larger than the polarization value at the maximum electric field, compared with the loop measured at $100 \mathrm{~Hz}$. This may be caused by $\mathrm{Mo}^{6+}$ and $\mathrm{Ti}^{4+}$ codoping, which degrades the resistivity [see Fig. 3(e)]. In comparison, $\mathrm{KNO}$ is rather insulating and an unlossy loop can be obtained at low frequencies, e.g., $1 \mathrm{~Hz}$. The FE hysteresis loops of KNO are relatively independent of frequency. Taking these factors into account, we estimate the true remanent polarization of the $90-10 \mathrm{KNTM}$ ceramics to be about $0.1 \mathrm{C} / \mathrm{m}^{2}$. This is smaller than the calculated values, most likely due to the increased measurement temperature ( $300 \mathrm{~K}$ in measurement and $0 \mathrm{~K}$ in calculation) and the polycrystalline microstructure (i.e., the existence of grain boundaries and domain walls suppress the FE switching). Moreover, the FE properties of 90-10KNTM are also likely to be suppressed by its smaller grain size than those of KNO (see Fig. S7 within the Supplemental Material).

\section{CONCLUSION}

To summarize, we use first-principles calculations to design $\mathrm{Mo}^{6+}$-containing perovskite KNTM and then carry out experimental synthesis and characterization to verify the predicted properties of this material. Consistent with theoretical predictions, it is found that this material exhibits ferroelectricity, a high ferroelectric-paraelectric transition temperature similar to that of the parent $\mathrm{KNO}$, and a low direct $E_{g}$ of less than $2.2 \mathrm{eV}$, despite only including $d^{0}$ metals that are traditionally considered to be unfavorable for obtaining visible-light absorption. Thus, this solid-solution system is promising for further studies of its optical, ferroelectric, and piezoelectric properties and demonstrates the utility of first-principles calculations for the exploration of new photoferroelectric materials.

\section{ACKNOWLEDGMENTS}

O.S. and I.G. acknowledge support by the US-Israel Binational Science Foundation (Grant No. 2016637). Y.B. would like to acknowledge joint funding by the University of Oulu and the Academy of Finland profiling action "Ubiquitous wireless sensor systems"' (Grant No. 24302332). The authors also acknowledge the Centre for Material Analysis of the University of Oulu for the use of their facilities.

[1] C. Paillard, X. Bai, I. C. Infante, M. Guennou, G. Geneste, M. Alexe, J. Kreisel, and B. Dkhil, Photovoltaics with ferroelectrics: Current status and beyond, Adv. Mater. 28, 5153 (2016). 
[2] P. Lopez-Varo, L. Bertoluzzi, J. Bisquert, M. Alexe, M. Coll, J. Huang, J. A. Jimenez-Tejada, T. Kirchartz, R. Nechache, F. Rosei, and Y. Yuan, Physical aspects of ferroelectric semiconductors for photovoltaic solar energy conversion, Phys. Rep.

[3] Y. Bai, H. Jantunen, and J. Juuti, Ferroelectric oxides for solar energy conversion, multi-source energy harvesting/sensing, and opto-ferroelectric applications, ChemSus Chem 12, 2540 (2019).

[4] J. E. Spanier, V. M. Fridkin, A. M. Rappe, A. R. Akbashev, A. Polemi, Y. Qi, Z. Gu, S. M. Young, C. J. Hawley, D. Imbrenda, G. Xiao, A. L. Bennett-Jackson, and C. L. Johnson, Power conversion efficiency exceeding the shockleyqueisser limit in a ferroelectric insulator, Nat. Photonics 10, 611 (2016).

[5] L. Wu, A. Podpirka, J. E. Spanier, and P. K. Davies, Ferroelectric, optical, and photovoltaic properties of morphotropic phase boundary compositions in the $\mathrm{PbTiO}_{3}$ $\mathrm{BiFeO} 3-\mathrm{Bi}\left(\mathrm{Ni}_{1 / 2} \mathrm{Ti}_{1 / 2}\right) \mathrm{O}_{3}$ system, Chem. Mater. 31, 4184 (2019).

[6] L. Wu, A. R. Akbashev, A. A. Podpirka, J. E. Spanier, and P. K. Davies, Infrared-to-Ultraviolet light-absorbing $\mathrm{BaTiO}_{3}$-based ferroelectric photovoltaic materials, J. Am. Ceram. Soc. 102, 4188 (2019).

[7] I. Grinberg, D. V. West, M. Torres, G. Gou, D. M. Stein, L. Wu, G. Chen, E. M. Gallo, A. R. Akbashev, P. K. Davies, J. E. Spanier, and A. M. Rappe, Perovskite oxides for visible-light-absorbing ferroelectric and photovoltaic materials, Nature 503, 509 (2013).

[8] Y. Bai, P. Tofel, J. Palosaari, H. Jantunen, and J. Juuti, A game changer: A multifunctional perovskite exhibiting giant ferroelectricity and narrow bandgap with potential application in a truly monolithic multienergy harvester or sensor, Adv. Mater. 29, 1 (2017).

[9] Y. Bai, T. Siponkoski, J. Peräntie, H. Jantunen, and J. Juuti, Ferroelectric, pyroelectric, and piezoelectric properties of a photovoltaic perovskite oxide, Appl. Phys. Lett. 110, 063903 (2017).

[10] H. Xiao, W. Dong, Y. Guo, Y. Wang, H. Zhong, Q. Li, and M.-M. Yang, Design for highly piezoelectric and visible/near-infrared photoresponsive perovskite oxides, Adv. Mater. 31, 1805802 (2019).

[11] T. Choi, S. Lee, Y. J. Choi, V. Kiryukhin, and S. W. Cheong, Switchable ferroelectric diode and photovoltaic effect in $\mathrm{BiFeO}_{3}$, Science (80-.) 324, 63 (2009).

[12] S. Y. Yang, J. Seidel, S. J. Byrnes, P. Shafer, C. H. Yang, M. D. Rossell, P. Yu, Y. H. Chu, J. F. Scott, J. W. Ager, L. W. Martin, and R. Ramesh, Above-Bandgap voltages from ferroelectric photovoltaic devices, Nat. Nanotechnol. 5, 143 (2010).

[13] S. Y. Yang, L. W. Martin, S. J. Byrnes, T. E. Conry, S. R. Basu, D. Paran, L. Reichertz, J. Ihlefeld, C. Adamo, A. Melville, Y. H. Chu, C. H. Yang, J. L. Musfeldt, D. G. Schlom, J. W. Ager, and R. Ramesh, Photovoltaic effects in $\mathrm{BiFeO}_{3}$, Appl. Phys. Lett. 95, 062909 (2009).

[14] M. Alexe and D. Hesse, Tip-Enhanced photovoltaic effects in bismuth ferrite, Nat. Commun. 2, 1 (2011).

[15] R. Nechache, C. Harnagea, S. Li, L. Cardenas, W. Huang, J. Chakrabartty, and F. Rosei, Bandgap tuning of multiferroic oxide solar cells, Nat. Photonics 9, 61 (2014).
[16] C. Pascual-Gonzalez, G. Schileo, S. Murakami, A. Khesro, D. Wang, I. M. Reaney, and A. Feteira, Continuously controllable optical band Gap in orthorhombic ferroelectric $\mathrm{KNbO}_{3}-\mathrm{BiFeO}_{3}$ ceramics, Appl. Phys. Lett. 110, 172902 (2017).

[17] O. Shafir, J. Yang, A. M. Rappe, and I. Grinberg, Ferroelectric barium titanate derivatives containing $\mathrm{Mo}$ and $\mathrm{Mg}$ for transparent photovoltaic applications, J. Appl. Phys. 126, 174101 (2019).

[18] K. M. Ok, P. S. Halasyamani, D. Casanova, M. Llunell, P. Alemany, S. Alvarez, and W. E. Geiger, Distortions in octahedrally coordinated d 0 transition metal oxides: A continuous symmetry measures approach, Chem. Mater. 18, 3176 (2006).

[19] See the Supplemental Material at http://link.aps.org/supple mental/10.1103/PhysRevApplied.14.044052 for additional information regarding the computationally studied structures, SEM images, XRD pattern of $\left(\mathrm{KNbO}_{3}\right)_{0.75}$ $\left(\mathrm{KTi}_{1 / 2} \mathrm{Mo}_{1 / 2} \mathrm{O}_{3}\right)_{0.25}$, and detailed $E_{g}$ determinations according to both the direct and indirect assumptions and band structures.

[20] P. Giannozzi, et al., QUANTUM ESPRESSO: A modular and open-source software project for quantum simulations of materials, J. Phys. Condens. Matter 21, 395502 (2009).

[21] R. G. Parr, S. R. Gadre, and L. J. Bartolotti, Local density functional theory of atoms and molecules, Proc. Natl. Acad. Sci. 76, 2522 LP (1979).

[22] J. P. Perdew and A. Zunger, Self-Interaction correction to density-functional approximations for many-electron systems, Phys. Rev. B 23, 5048 (1981).

[23] D. I. Bilc, R. Orlando, R. Shaltaf, G. M. Rignanese, J. Íñiguez, and P. Ghosez, Hybrid exchange-correlation functional for accurate prediction of the electronic and structural properties of ferroelectric oxides, Phys. Rev. B: Condens. Matter Mater. Phys. 77, 1 (2008).

[24] Z. Wu and R. E. Cohen, More accurate generalized gradient approximation for solids, Phys. Rev. B: Condens. Matter Mater. Phys. 73, 235116 (2006).

[25] D. Vanderbilt and M. H. Cohen, Monoclinic and triclinic phases in higher-order devonshire theory, Phys. Rev. B: Condens. Matter Mater. Phys. 63, 94108 (2001).

[26] B. P. Burton and E. Cockayne, Why $\mathrm{Pb}\left(\mathrm{B}, \mathrm{B}^{\prime}\right) \mathrm{O}_{3}$ perovskites disorder at lower temperatures than $\mathrm{Ba}\left(\mathrm{B}, \mathrm{B}^{\prime}\right) \mathrm{O}_{3}$ perovskites, Phys. Rev. B 60, R12542 (1999).

[27] S. C. Abrahams, S. K. Kurtz, and P. B. Jamieson, Atomic displacement relationship to curie temperature and spontaneous polarization in displacive ferroelectrics, Phys. Rev. 172, 551 (1968).

[28] I. Grinberg and A. M. Rappe, Local structure and macroscopic properties in $\mathrm{PbMg}_{1 / 3} \mathrm{Nb}_{2 / 3} \mathrm{O}_{3}-\mathrm{PbTiO}_{3}$ and $\mathrm{PbZn}_{1 / 3} \mathrm{Nb}_{2 / 3} \mathrm{O}_{3}-\mathrm{PbTiO}_{3}$ solid solutions, Phys. Rev. B: Condens. Matter Mater. Phys. 70, 220101 (2004).

[29] H. Birol, D. Damjanovic, and N. Setter, Preparation and characterization of $\mathrm{KNbO}_{3}$ ceramics, J. Am. Ceram. Soc. 88, 1754 (2005).

[30] R. López and R. Gómez, Band-Gap energy estimation from diffuse reflectance measurements on Sol-Gel and commercial $\mathrm{TiO}_{2}$ : A comparative study, J. Sol-Gel Sci. Technol. 61, 1 (2012). 